\title{
Density Functional Investigations on the C-C Bond Formation and Cleavage in Molecular Batteries
}

\author{
Paola Belanzoni, Marzio Rosi, and Antonio Sgamellotti \\ Istituto di Scienze e Tecnologie Molecolari del CNR, c/o Dipartimento di Chimica, \\ Università di Perugia, \\ Via Elce di Sotto, 806123 Perugia, Italy \\ paola@thch.unipg.it
}

\begin{abstract}
Density functional calculations have been performed on titanium, nickel, molybdenum and niobium Schiff base complexes and titanium, nickel porphyrinogen complexes in order to understand the behaviour of these systems in redox processes. In titanium and nickel Schiff base complexes C-C $\sigma$ bonds are formed upon reduction, while in titanium and nickel porphyrinogen complexes C-C $\sigma$ bonds are formed upon oxidation. In both systems, the formation or the cleavage of C-C bonds avoids a variation in the oxidation state of the metal and these $\mathrm{C}-\mathrm{C}$ bonds act not only as electron reservoirs, but also as a buffer for the oxidation state of the metal. In the molybdenum Schiff base complexes a preferential formation of metal-metal bonds upon reduction is calculated, while in the niobium analogues the formation of $\mathrm{C}-\mathrm{C}$ bonds competes with that of M-M bonds, the latter being the first ones to be involved in electron-transfer reactions.
\end{abstract}

\section{Introduction}

The use of a chemical bond across two atoms for the storage and subsequent release of a pair of electrons seems to be quite an obvious concept. The reductive or oxidative coupling and the reverse decoupling can be the mechanism through which we must pass through [1]. Many mechanisms are known which operate in at least one direction $[2,3]$, while very often the reverse process does not even occur. A major question is under which conditions can we take advantage of a simple chemical bond for storing and releasing electrons. The major unquestionable goal is the facile reversibility associated with no overall change when the chemical system is returned to its original state. This is a normal event when the redox reactions involving formation and cleavage of a chemical bond are performed electrochemically [4]. However, reversibility to the original state is much more rarely observed when the redox system is involved in the exchange of electrons with chemical substrates [5]. As a matter of fact, the exchange of electrons with the substrate usually occurs at centres which function as electron reservoirs, so that the substrate remains bonded to them [6]. 
With the perspective of the use of chemical bonds as electron reservoirs, we selected the formation and cleavage of $\mathrm{C}-\mathrm{C}$ single bonds as suitable candidates for storing and releasing a pair of electrons. Moreover, we tried to put metal-metal and carbon-carbon bonds in competition within the same molecular framework. The present paper is focused on density functional calculations on suitable model systems, performed in order to understand how $\mathrm{C}-\mathrm{C}$ units can function as electron shuttles in redox processes, the role played by the metal and the requisites for the reversibility of the electron storage.

\section{Computational Details}

\subsection{Model Systems}

We can distinguish between two main classes of compounds: i) transition metal Schiff base complexes, in which the $\mathrm{C}-\mathrm{C} \sigma$ bond arises from a reductive process, as depicted in Figure 1, and ii) porphyrinogen complexes, in which the $\mathrm{C}-\mathrm{C} \sigma$ bond originates from an oxidative process, as shown in Figure 2.
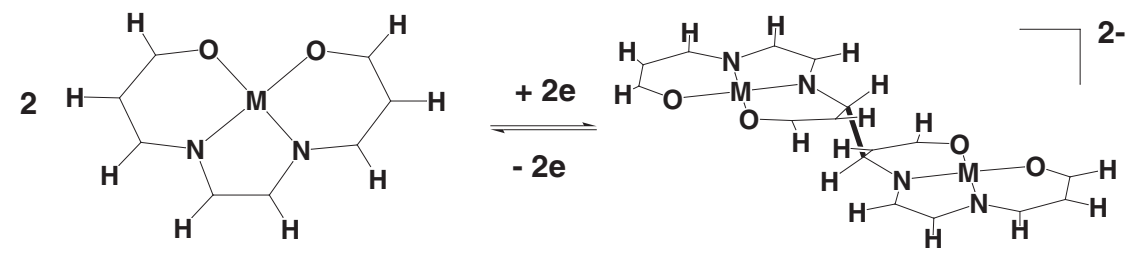

Fig. 1. C-C $\sigma$ bond formation upon a reductive process
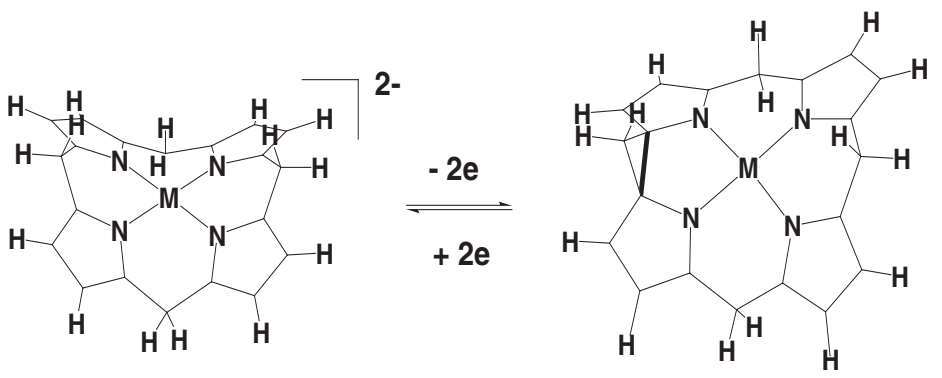

Fig. 2. C-C $\sigma$ bond formation upon an oxidative process

For the first class of complexes, $[\mathrm{Ni}($ salophen)] [salophen $\equiv \mathrm{N}, \mathrm{N}$ ' - phenylenebis(salicylideneaminato)dianion], [Ti(salophen) $\left.\mathrm{Cl}_{2}\right],[\mathrm{Ti}$ (salophen $\left.)\right]_{2}^{2+}$ and, $[\mathrm{M}(\text { salophen })]_{2}$ $(\mathrm{M}=\mathrm{Mo}, \mathrm{Nb})$ are considered as models for the oxidized form of transition metal Schiff base complexes, while $\left[\mathrm{Ti}_{2}\left({ }^{*} \text { salophen }_{2}{ }^{*}\right)\right]^{2-}$ and $\left[\mathrm{M}_{2}\left({ }^{*}\right.\right.$ salophen $\left.\left._{2}{ }^{*}\right)\right](\mathrm{M}=\mathrm{Mo}, \mathrm{Nb})$, 
in which ${ }^{*}$ salophen $_{2}{ }^{*}$ is the octadentate, octaanionic ligand derived by a fourelectron reduction of two salophen ligands, are considered as model of reduced, imino-coupled, transition metal Schiff base complexes. For the second class of complexes, $\left[\mathrm{TiH}_{8} \mathrm{~N}_{4}\right]$ and $[\mathrm{Ni}(\text { porphyrinogen })]^{2-}$ are considered as model for the reduced form of a porphyrinogen complex.

In order to make the calculations feasible, some simplifications have been made in the investigated systems. In particular, in the salophen ligands the aromatic rings were substituted by $\mathrm{C}=\mathrm{C}$ double bonds, while in porphyrinogen the ethyl meso groups were replaced by hydrogen atoms, as shown in Figure 3.

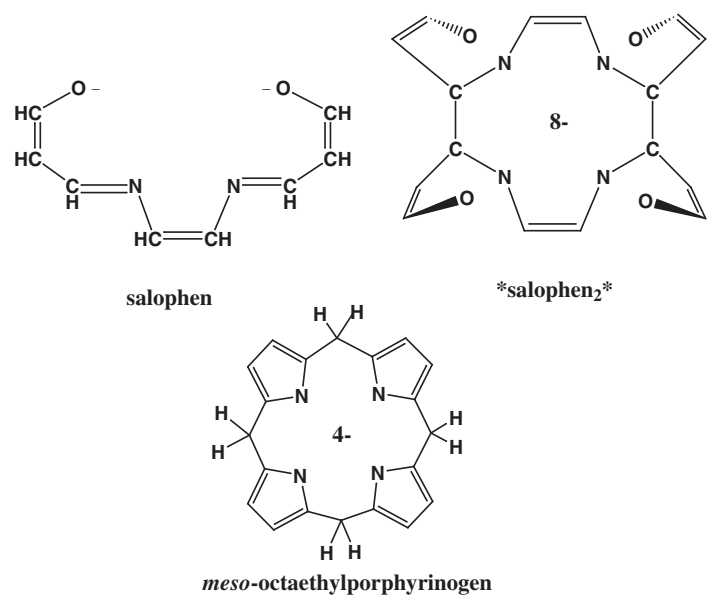

Fig. 3. Model representation of the salophen and meso-octaethylporphyrinogen ligands used in the calculations

These simplifications should not affect significantly the electronic distribution around the transition metal and the $\mathrm{C}-\mathrm{C} \sigma$ bond formation and breaking mechanisms.

\subsection{Methods}

Density functional theory (DFT) has been used for the determination of equilibrium geometries and the evaluation of the energetics of all the investigated systems and processes. The BP86 exchange-correlation functional was used for all the calculations. This functional is based on the Becke's nonlocal exchange [7] and Perdew nonlocal correlation $[8,9]$ corrections to the local density approximation. Open shell systems have been calculated with the spin unrestricted approach. The calculations have been performed using the ADF (Amsterdam Density Functional) program package [10] (Gaussian 94 program package [11] for titanium systems) and were done on a cluster of IBM RISC/6000 workstations and on IBM SP3. The atomic charges have been obtained through a Mulliken population analysis. 


\subsection{Basis Sets}

For the ADF calculations the molecular orbitals were expanded in an uncontracted double- $\zeta$ STO basis set for all atoms with the exception of $3 \mathrm{~d}$ and $4 \mathrm{~s}$ $\mathrm{Ni}$ orbitals, and of $4 \mathrm{~d}$ and $5 \mathrm{~s}$ Mo and $\mathrm{Nb}$ orbitals for which we used a triple- $\zeta$ STO basis set. This basis set was augmented by one $4 \mathrm{p}$ function for Ni, two $5 \mathrm{p}$ functions for Mo and $\mathrm{Nb}$, one $2 \mathrm{p}$ polarization function for hydrogen and one $3 \mathrm{~d}$ polarization function for the other elements. The cores (Ni: 1s-2p; Mo,Nb: 1s-3d; C,N,O: 1s) have been kept frozen. The basis set employed for the Gaussian 94 calculations on the titanium-Schiff base complexes is based on the WachtersHay set $[12,13]$ for the transition metal atom and on the $6-31 G^{*}$ set [14] for all the other atoms, except chlorine, which was described with a 6 -31G set [14]. The same basis set, with the addition of diffuse functions [15] on nitrogen, was used for most of the calculations performed on the titanium-porphyrinogen complexes. Due to the size of these systems, however, some geometric optimizations of these complexes were performed using a smaller set, based on the WachtersHay set $[12,13]$ for the transition metal atom, the 6-31G set [14] for nitrogen and the $3-21 \mathrm{G}$ set [16] for carbon and hydrogen. Only the spherical harmonic components of the basis sets were used.

\subsection{Geometry Optimization}

The geometry of the model systems considered was fully optimized starting from parameters deduced from the available experimental X-ray structures $[17-$ 19]. We considered $\mathrm{C}_{2 v}$ symmetry for [ $\mathrm{Ti}$ (salophen) $\left.\mathrm{Cl}_{2}\right]$ and [ $\mathrm{Ni}$ (salophen)], $\mathrm{C}_{i}$ symmetry for $[\mathrm{Ti}(\text { salophen })]_{2}^{2+},[\mathrm{Ni}(\text { salophen })]_{2}^{2-},\left[\mathrm{Ti}_{2}\left({ }^{*} \text { salophen }_{2}{ }^{*}\right)\right]^{2-}$ and $\left[\mathrm{M}_{2}\left({ }^{*}\right.\right.$ salophen $\left.\left._{2}{ }^{*}\right)\right] \mathrm{M}=\mathrm{Mo}, \mathrm{Nb}, \mathrm{C}_{2 h}$ symmetry for $[\mathrm{M} \text { (salophen) }]_{2} \mathrm{M}=\mathrm{Mo}, \mathrm{Nb}, \mathrm{C}_{2}$ symmetry for $[\mathrm{Ni}(\text { porphyrinogen })]^{2-}$ and $\mathrm{C}_{s}$ symmetry for $[\mathrm{Ni}($ porphyrinogen $)(\Delta)]$. The counterions were not considered explicitely in the model systems. For the titanium-porphyrinogen complexes, first we fully optimized the geometry of $\left[\mathrm{H}_{8} \mathrm{~N}_{4}\right]^{4-}$ and $\left[\mathrm{H}_{8} \mathrm{~N}_{4}(\Delta)_{2}\right]$, where $\left[\mathrm{H}_{8} \mathrm{~N}_{4} \mathrm{H}_{4}\right]$ is the porphyrinogen and $\Delta$ denotes a cyclopropane unit, in order to compare the reduced and oxidized forms of the free ligand. Starting from this optimized geometry for the ligands, we subsequently optimized the geometry of the titanium complexes. We considered both the species $\left[\mathrm{M}(\mathrm{IV}) \mathrm{H}_{8} \mathrm{~N}_{4}\right]$ and $\left[\mathrm{M}(0) \mathrm{H}_{8} \mathrm{~N}_{4}(\Delta)_{2}\right]$, in order to analyze the relative stability. The geometry optimizations of the free ligands were performed with the larger basis set, while those of the complexes were performed with the smaller basis set; for the titanium complexes, however, we computed the energies at the optimized geometries also with the larger basis set. The optimizations were performed considering an $\mathrm{S}_{4}$ symmetry for $\left[\mathrm{MH}_{8} \mathrm{~N}_{4}\right]$ and a $\mathrm{C}_{2}$ symmetry for $\left[\mathrm{MH}_{8} \mathrm{~N}_{4}(\Delta)_{2}\right]$. 


\section{Results and Discussion}

\subsection{Titanium Schiff Base and Titanium Porphyrinogen Complexes}

The optimized structure of $\left[\mathrm{Ti}(\right.$ salophen $\left.) \mathrm{Cl}_{2}\right]$ shows an octahedral coordination around the transition metal, while the optimized geometry of the dimeric species $[\mathrm{Ti}(\text { salophen })]_{2}{ }^{2+}$ shows a square pyramidal coordination around the metal. The main features of the optimized structure of $\left[\mathrm{Ti}_{2}\left({ }^{*} \operatorname{salophen}_{2}{ }^{*}\right)\right]^{2-}$ are a clear rearrangement of the geometry of the salophen ligands, the presence of two C-C bonds $(1.618 \AA$ ) between carbon atoms of imino groups of the two original salophen ligands, and the presence of an interaction between the two metal centers, the Ti-Ti distance being $2.452 \stackrel{\circ}{A}$. The ground state of $[\mathrm{Ti}$ (salophen $\left.) \mathrm{Cl}_{2}\right]$ is the singlet ${ }^{1} \mathrm{~A}_{1}$, and the highest occupied molecular orbital (HOMO) is mainly localized on the salophen ligand. The lowest unoccupied molecular orbital (LUMO) and the orbitals immediately at higher energy have mainly titanium d character, with small $\mathrm{C}=\mathrm{N} \pi$-antibonding components. The ground state of $[\mathrm{Ti}(\text { salophen })]_{2}{ }^{2+}$ is the triplet ${ }^{3} \mathrm{~A}_{u}$, with the two singly occupied orbitals essentially metal in character. The LUMO and the orbitals immediately above are mainly bonding and antibonding combinations of $\mathrm{d}$ orbitals of the two Ti atoms, which do not interact at all between them. In both the model systems we have considered for the oxidized species, a reductive process should involve the metal, with a change in its oxidation state, since the LUMO is mainly metal $\mathrm{d}$ in character, at least in so far as the structure of the complex does not change. The ground state of the reduced species $\left[\mathrm{Ti}_{2}\left({ }^{*} \text { salophen }_{2}{ }^{*}\right)\right]^{2-}$ is the singlet ${ }^{1} \mathrm{~A}_{g}$, and the HOMO represents a $\sigma$ bond between the two metal centers. The orbitals immediately lower in energy are mainly ligand in character and the $\mathrm{C}-\mathrm{C} \sigma$ bonds of the imino groups of the two different salophen units lie at even lower energy. The LUMO is a bonding combination of metal d orbitals. An oxidative process of this system involves in the first place the metal centers and should cause a change in the oxidation state of the metal. However, the charge on the Ti atoms does not differ appreciably between the oxidized species $[\mathrm{Ti} \text { (salophen) }]_{2}{ }^{2+}$ and the reduced species $\left[\mathrm{Ti}_{2}\left({ }^{*} \text { salophen }_{2}{ }^{*}\right)\right]^{2-}$. Therefore, we can say that in the redox process the metal is the first species that acquires or loses electrons, but the geometry rearrangement of the system is able to buffer the variation in the number of the electrons and to maintain an unchanged oxidation state of the metal.

The optimized geometry of the free porphyrinogen ligand $\left[\mathrm{H}_{8} \mathrm{~N}_{4}\right]^{4-}$ has a very distorted structure that allows the negatively charged $\mathrm{N}$ atoms to be as far away as possible from each other. The $\left.\left[\mathrm{H}_{8} \mathrm{~N}_{4}(\Delta)_{2}\right)\right]$ ligand has a calix structure, with the two cyclopropane rings pointing away from the cavity of the system. Energetically, the reduced species is strongly destabilized, so that the redox cycle shown in Figure 4 does not seem to be possible for the free ligands. The inclusion of a $\mathrm{Ti}^{4+}$ ion interacting with the ligands gives rise to an almost planar structure for $\left[\mathrm{TiH}_{8} \mathrm{~N}_{4}\right]$ system and to a structure for $\left[\mathrm{TiH}_{8} \mathrm{~N}_{4}(\Delta)_{2}\right]^{4+}$ very similar to that of the free ligand, with the metal located on top of the calix. The reduced species in this case is much more stable than the oxidized one, as expected. 


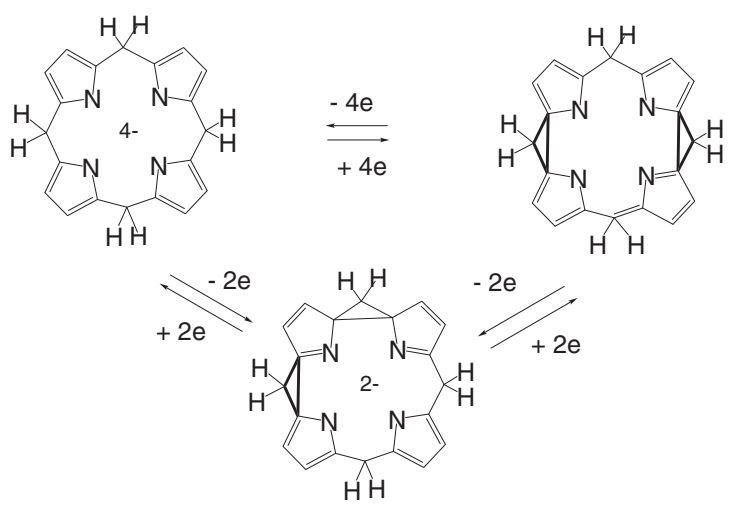

Fig. 4. C-C $\sigma$ bond formation upon an oxidative process

From our calculations we can say that titanium prefers high oxidation states (IV) in the presence of ligands like porphyrinogen that are able to accept electrons. With a metal that needs electrons, porphyrinogen assumes the structure with two cyclopropane units and it is able to donate up to four electrons, while with a metal that can be easily oxidized porphyrinogen accepts up to four electrons. We conclude that the presence of the porphyrinogen coordinated to a metal has a buffering effect on the oxidation state of the metal. The only requirement is that electrons must be exchanged in couples; the removal of each couple corresponds to the formation of a cyclopropane unit on the porphyrinogen skeleton.

\subsection{Nickel Schiff Base and Nickel Porphyrinogen Complexes}

The optimized structure of the oxidized form of the nickel Schiff base model system $[\mathrm{Ni}($ salophen)] shows a planar coordination around the transition metal. The reduction by two electrons of the monomeric species gives rise, through the reductive coupling of the imino groups, to the dimeric species $[\mathrm{Ni} \text { (salophen) }]_{2}{ }^{2-}$. The reduction does not affect significantly the coordination around the metal, which, as it is suggested by the Mulliken population analysis, does not change its oxidation state. The main variation upon reduction is the formation of a $\mathrm{C}-\mathrm{C}$ $\sigma$ bond of $1.617 \AA$ between carbon atoms of imino groups of the two original salophen ligand. In the optimized structure of $[\mathrm{Ni}(\text { porphyrinogen })]^{2-}$ species, the presence of the metal in the middle of the $\mathrm{N}_{4}$ core of the porphyrinogen gives rise to an almost planar structure. The oxidation by two electrons of this species gives rise to $[\mathrm{Ni}($ porphyrinogen $)(\Delta)]$, whose optimized structure does not show significant variations in the coordination of the nickel, while shows a strong rearrangement in the ligand, leading to the formation of a cyclopropane ring in it. Also in this case we do not have a variation in the oxidation state of the metal, the oxidation being localized on the ligand through the formation of a C-C $\sigma$ bond of $1.613 \stackrel{\circ}{A}$. [Ni(salophen)] and [Ni(porphyrinogen) $(\Delta)]$ show therefore a complementary behaviour upon redox processes: the first system 
indeed gives rise to the formation of $\mathrm{C}-\mathrm{C} \sigma$ bonds upon reduction, while the second one shows a similar behaviour upon oxidation. Moreover, [Ni(salophen)] (1) and $[\mathrm{Ni}(\text { porphyrinogen })]^{2-}(\mathbf{3})$ can be seen as the partners of a redox reaction, whose products are $[\mathrm{Ni}(\text { salophen })]_{2}^{2-}(\mathbf{2})$ and $[\mathrm{Ni}($ porphyrinogen $)(\Delta)](\mathbf{4})$ :

$$
2 \times(\mathbf{1})+(3) \rightarrow(2)+(4)
$$

The reverse of this reaction is reported in Figure 5.
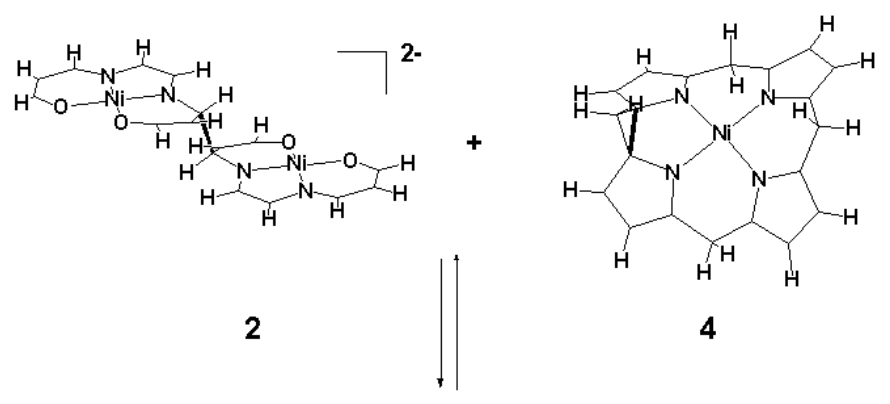

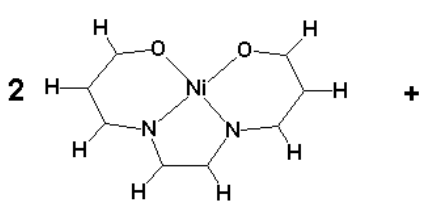

1

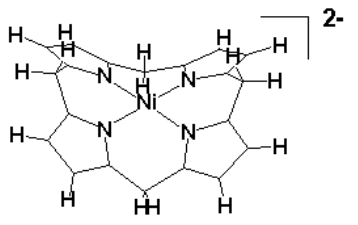

3

Fig. 5. An electrochemical cell based on C-C bonds breaking

From the calculated total energies of the systems, this reaction is computed to be exothermic and its $\Delta \mathrm{E}$ is equal to $-275.9 \mathrm{~kJ} \mathrm{~mol}^{-1}$. Assuming $\Delta \mathrm{G} \simeq \Delta \mathrm{E}$, a rough estimate of the standard voltage of this redox reaction is $\Delta \mathrm{V} \simeq 1.4 \mathrm{~V}$. The above reaction can be considered in an electrochemical cell, whose functioning is based on C-C bond breaking. Half-reaction $(\mathbf{4}) \rightarrow(3)$ is the cathode of the cell, while half-reaction $(\mathbf{2}) \rightarrow 2 \times(\mathbf{1})$ is the anode of the cell. While the discharge of the cell implies $\mathrm{C}-\mathrm{C}$ bonds cleavage, its recharge gives rise to formation of $\mathrm{C}-\mathrm{C}$ bonds.

\subsection{Molybdenum and Niobium Schiff Base Complexes}

The optimized structure of $[\mathrm{Mo}(\text { salophen })]_{2}$ shows a Mo-Mo bond length of 2.195 $\stackrel{\circ}{ }$ which is typical of a Mo-Mo quadruple bond, that is expected for a Mo (II) complex. A metal-to-ligand four-electron transfer in the Mo (II) complex $[\mathrm{Mo}(\text { salophen })]_{2}$ would give rise to the Mo (IV) complex $\left[\mathrm{Mo}_{2}\left({ }^{*} \operatorname{salophen}_{2}{ }^{*}\right)\right]$, whose optimized geometry shows a Mo-Mo bond length of $2.538 \stackrel{\circ}{A}$, very close 
to a Mo-Mo double bond, and the presence of two $\mathrm{C}-\mathrm{C} \sigma$ bonds between carbon atoms of the imino groups of the two salophen ligands. $\left[\mathrm{Mo}_{2}\left({ }^{*}\right.\right.$ salophen $\left.\left._{2}{ }^{*}\right)\right]$ is computed to be less stable than $[\mathrm{Mo}(\text { salophen })]_{2}$ by $295.0 \mathrm{~kJ} \mathrm{~mol}^{-1}$. In the optimized structure of $[\mathrm{Nb} \text { (salophen) }]_{2}$, the calculated $\mathrm{Nb}-\mathrm{Nb}$ bond length $(2.433 \AA$ ) is typical of an Nb-Nb triple bond, as expected for an Nb (II) complex. A metalto-ligand four-electron transfer in the $\mathrm{Nb}$ (II) complex [Nb(salophen) $]_{2}$ would give rise to the $\mathrm{Nb}(\mathrm{IV})$ complex $\left[\mathrm{Nb}_{2}\left({ }^{*}\right.\right.$ salophen $\left.\left._{2}{ }^{*}\right)\right]$, whose optimized geometry shows a $\mathrm{Nb}-\mathrm{Nb}$ bond length of $2.625 \stackrel{\circ}{A}$, very close to a $\mathrm{Nb}-\mathrm{Nb}$ single bond, and the presence of two C-C $\sigma$ bonds between carbon atoms of the imino groups of the two salophen ligands. $\left[\mathrm{Nb}_{2}\left({ }^{*}\right.\right.$ salophen $\left.\left._{2}{ }^{*}\right)\right]$ is computed to be less stable than $[\mathrm{Nb}(\text { salophen })]_{2}$ by only $52.3 \mathrm{~kJ} \mathrm{~mol}{ }^{-1}$. For $\left[\mathrm{Nb}_{2}\left({ }^{*}\right.\right.$ salophen $\left.\left._{2}{ }^{*}\right)\right]$ complex, the HOMO orbital essentially describes a Nb-Nb $\sigma$ bond, while the LUMO orbital can be mainly characterized as a bonding $\delta$ orbital. The next orbital above the LUMO has mainly Nb-Nb $\pi$ bonding character. The next valence orbitals at lower energies with respect to the HOMO are mainly composed of ligand atoms orbitals, while the orbitals describing the two $\mathrm{C}-\mathrm{C} \sigma$ bonds between carbon atoms of imino groups of the two salophen ligands lie at lower energies. An oxidation of $\left[\mathrm{Nb}_{2}\left({ }^{*}\right.\right.$ salophen $\left.\left.{ }_{2}^{*}\right)\right]$, therefore, should affect only the $\mathrm{Nb}-\mathrm{Nb}$ bond and not the two C-C $\sigma$ bonds. Both $\left[\mathrm{Nb}_{2}\left({ }^{*}\right.\right.$ salophen $\left.\left._{2}{ }^{*}\right)\right]$ and $[\mathrm{Nb}(\text { salophen })]_{2}$ can formally give rise to different levels of electron storage. The electron reservoirs are the $\mathrm{C}-\mathrm{C} \sigma$ bonds between the carbon atoms of the imino groups of the two salophen ligands or the metal-metal bonds in the $\left[\mathrm{Nb}_{2}\left({ }^{*}\right.\right.$ salophen $\left.\left._{2}{ }^{*}\right)\right]$ species, while only metal-metal bonds can act as electron reservoirs in $[\mathrm{Nb} \text { (salophen) }]_{2}$ complexes. In order to analyze this point we investigated, in addition to the neutral species, the $2-, 2+, 4+$ and $6+$ species, which allow the storage or release of up to eight electrons (Fig. 6).

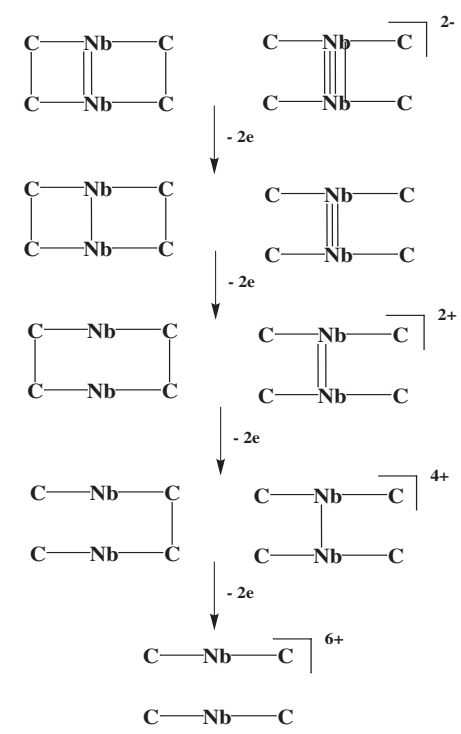

Fig. 6. Different levels of electrons storage 
The optimized geometry of $\left[\mathrm{Nb}_{2}\left({ }^{*} \text { salophen }_{2}{ }^{*}\right)\right]^{2-}$ shows two C-C $\sigma$ bonds between the carbon atoms of the imino groups of the two salophen ligands and the presence of a double $(\sigma$ and $\delta$ ) bond between the two metal centres. The neutral compound shows a single Nb-Nb bond and two C-C $\sigma$ bonds. A two-electron oxidation of this species implies the cleavage of the $\mathrm{Nb}-\mathrm{Nb}$ bond, and the removal of another two electrons implies the breaking of one $\mathrm{C}-\mathrm{C} \sigma$ bond and, finally, the removal of the next two electrons gives rise to two units which do not interact at all. With regard to the $[\mathrm{Nb} \text { (salophen) }]_{2}{ }^{x}$ species (where $x=2-, 0,2+, 4+, 6+$ ), we have a $\mathrm{Nb}-\mathrm{Nb}$ quadruple bond in the dianion, a triple bond in the neutral species, a double bond in the dication, a single bond in the $4+$ species and a lack of any metal-metal interaction in the $6+$ species. In the two analysed series of compounds, the metal-metal bonds are always preferred with respect to carbon-carbon bonds.

\section{Conclusion}

The present study at density functional level has shown that titanium, nickel Schiff base complexes and titanium, nickel porphyrinogen complexes show similar, although complementary, behaviour in redox processes. In transition metal Schiff base complexes C-C $\sigma$ bonds are formed upon acquisition of pairs of electrons, while in transition metal porphyrinogen complexes $\mathrm{C}-\mathrm{C} \sigma$ bonds are formed upon loss of pairs of electrons. In both systems, the formation or the breaking of the $\mathrm{C}-\mathrm{C}$ bonds avoids a variation in the oxidation state of the metal. These C-C bonds, therefore, act not only as electrons reservoirs, but also as a buffer of the oxidation state of the metal. The lack of variation in the oxidation state of the metal is the first step towards the reversibility of the redox process. The complementary behaviour in redox processes of nickel Schiff base complexes and nickel porphyrinogen complexes can be considered in a reversible electrochemical cell with the discharge process based on $\mathrm{C}-\mathrm{C}$ bonds breaking and the recharge based on $\mathrm{C}-\mathrm{C}$ bonds formation. The evaluation of the total energies of the investigated systems suggests that such an electrochemical cell presents an electrochemical potential suitable for practical applications. In molybdenum Schiff base complexes, a preferential formation of metal-metal bonds upon reduction is calculated, while in niobium analogues the formation of $\mathrm{C}-\mathrm{C}$ bonds competes with that of M-M bonds, the latter being the first ones to be involved in electron-transfer reactions.

\section{References}

1. Savéant, J.-M.: Electron Transfer, Bond Breaking and Bond Formation. In: Tidwell, T.T. (eds.): Advances in Physical Organic Chemistry, Vol. 35. Academic Press, San Diego (2000) 117-192

2. Hartley, F.R., Patai, S. (eds.): Carbon-Carbon Bond Formation Using Organometallic Compounds. Wiley, Chichester (1985)

3. Cotton, F.A., Wilkinson, G., Murillo, C.A., Bochmann, M.: Advanced Inorganic Chemistry, 6th ed. Wiley, New York (1999) 957-962 and references therein 
4. Baik, M.-H., Ziegler, T., Schauer, C.K.: Density Functional Theory Study of Redox Pairs. 1.Dinuclear Iron Complexes That Undergo Multielectron Redox Reactions Accompanied by a Reversible Structural Change. J. Am. Chem. Soc. 122 (2000) 9143-9154 and references therein

5. Rathore, R., Le Magueres, P., Lindeman, S.V., Kochi, J.K.: A Redox-Controlled Molecular Switch Based on the Reversible C-C Bond Formation in Octamethoxytetraphenylene. Angew. Chem. Int. Ed. 39 (2000) 809-812 and references therein

6. Rathore, R., Kochi, J.K.: Donor/Acceptor Associations and Electron-Transfer Paradigm in Organic Reactivity. In: Tidwell, T.T. (eds.): Advances in Physical Organic Chemistry, Vol. 35. Academic Press, San Diego (2000) 192-318 and references therein

7. Becke, A.D.: Density-Functional Exchange-Energy Approximation with Correct Asymptotic Behavior. Phys. Rev. A 38 (1988) 3098-3100

8. Perdew, J.P.: Density-Functional Approximation for the Correlation Energy of the Inhomogeneous Electron Gas. Phys. Rev. B 33 (1986) 8822-8824

9. Perdew, J.P.: Erratum Phys. Rev. B 34 (1986) 7406

10. ADF Program System Release 2.3.0 http://www.scm.com

11. Frisch, M.J., Trucks, G.W., Schlegel, H.B., Gill, P.M.W., Johnson, B.G., Robb, M.A., Cheeseman, J.R., Keith, T.A., Petersson, G.A., Montgomery, J.A., Raghavachari, K., Al-Laham, M.A., Zakrzewski, V.G., Ortiz, J.V., Foresman, J.B., Cioslowski, J., Stefanov, B.B., Nanayakkara, A., Challacombe, M., Peng, C.Y., Ayala, P.Y., Chen, W., Wong, M.W., Andres, J.L., Replogle, E.S., Gomperts, R., Martin, R.L., Fox, D.J., Binkley, J.S., Defrees, D.J., Baker, J., Stewart, J.P., HeadGordon, M., Gonzalez, C., Pople, J.A.: Gaussian 94, Gaussian, Inc., Pittsburgh, PA, 1995

12. Wachters, A.J.H.: Gaussian Basis Set for Molecular Wavefunctions Containing Third-Row Atoms. J. Chem. Phys. 52 (1970) 1033-1036

13. Hay, P.J.: Gaussian Basis Sets for Molecular Calculations. The Representation of 3d Orbitals in Transition Metal Atoms. J. Chem. Phys. 66 (1977) 4377-4384

14. Frisch, M.J., Pople, J.A., Binkley, J.S.: Self-Consistent Molecular Orbital Methods. 25.Supplementary Functions for Gaussian Basis Sets. J. Chem. Phys. 80 (1984) 3265-3269 and references therein

15. Clark, T., Chandrasekhar, J., Spitznagel, G.W., Schleyer, P. von R.: Efficient Diffuse Function-Augmented Basis Sets for Anion Calculations. III.The 3-21G Basis Set for First-Row Elements, Lithium to Fluorine. J. Comput. Chem. 4 (1983) 294-301

16. Binkley, J.S., Pople, J.A., Hehre, W.J.: Self-Consistent Molecular Orbital Methods. 21.Small Split-Valence Basis Sets for First-Row Elements. J. Am. Chem. Soc. 102 (1980) 939-947

17. Franceschi, F., Solari, E., Floriani, C., Rosi, M., Chiesi-Villa A., Rizzoli, C.: Molecular Batteries Based on Carbon-Carbon Bond Formation and Cleavage in Titanium and Vanadium Schiff Base Complexes. Chem. Eur. J. 5 (1999) 708-721

18. Franceschi, F., Solari, E., Scopelliti, R., Floriani, C.: Metal-Mediated Transfer of Electrons between Two Different C-C Single Bonds That Function as ElectronDonor and Electron-Acceptor Units. Angew. Chem. Int. Ed. 39 (2000) 1685-1687

19. Floriani, C., Solari, E., Franceschi, F., Scopelliti, R., Belanzoni, P., Rosi, M.: MetalMetal and Carbon-Carbon Bonds as Potential Components of Molecular Batteries. Chem. Eur. J. 7 (2001) 3052-3061 